\title{
Visualization of Aspalathin in Rooibos (Aspalathus linearis) Plant and Herbal Tea Extracts Using Thin-Layer Chromatography
}

\author{
Emily Amor Stander ${ }^{1,+}{ }^{+}$, Wesley Williams ${ }^{1,+}$, Fanie Rautenbach ${ }^{2}$, Marilize Le Roes-Hill ${ }^{3}{ }^{(0)}$, \\ Yamkela Mgwatyu ${ }^{1}$, Jeanine Marnewick ${ }^{2}$ and Uljana Hesse ${ }^{1,4, *}$ (i) \\ 1 South African National Bioinformatics Institute (SANBI), University of the Western Cape, Robert Sobukwe \\ Road, Bellville 7535, South Africa; emily.amor.stander@gmail.com (E.A.S.); wesleywt@gmail.com (W.W.); \\ yamkelamgwatyu@gmail.com (Y.M.) \\ 2 Oxidative Stress Research Centre, Faculty of Health and Wellness Sciences, Institute of Biomedical and \\ Microbial Biotechnology, Cape Peninsula University of Technology, Symphony Road, Bellville 7535, \\ South Africa; rautenbachf@cput.ac.za (F.R.); MarnewickJ@cput.ac.za (J.M.) \\ 3 Biocatalysis and Technical Biology Research Group, Institute of Biomedical and Microbial Biotechnology, \\ Cape Peninsula University of Technology, Symphony Road, Bellville 7535, South Africa; \\ LeRoesM@cput.ac.za \\ 4 Institute for Microbial Biotechnology and Metagenomics, University of the Western Cape, Robert Sobukwe \\ Road, Bellville 7535, South Africa \\ * Correspondence: uljana@sanbi.ac.za; Tel.: +27-(0)21-959-3573 \\ + Authors contributed equally to the work.
}

Received: 28 November 2018; Accepted: 18 January 2019; Published: 7 March 2019

\begin{abstract}
Aspalathin, the main polyphenol of rooibos (Aspalathus linearis), is associated with diverse health promoting properties of the tea. During fermentation, aspalathin is oxidized and concentrations are significantly reduced. Standardized methods for quality control of rooibos products do not investigate aspalathin, since current techniques of aspalathin detection require expensive equipment and expertise. Here, we describe a simple and fast thin-layer chromatography (TLC) method that can reproducibly visualize aspalathin in rooibos herbal tea and plant extracts at a limit of detection (LOD) equal to $178.7 \mathrm{ng}$ and a limit of quantification (LOQ) equal to $541.6 \mathrm{ng}$. Aspalathin is a rare compound, so far only found in A. linearis and its (rare) sister species A. pendula. Therefore, aspalathin could serve as a marker compound for authentication and quality control of rooibos products, and the described TLC method represents a cost-effective approach for high-throughput screening of plant and herbal tea extracts.
\end{abstract}

Keywords: Rooibos; Aspalathus linearis; aspalathin; thin-layer chromatography; p-anisaldehyde; herbal tea; authentication; marker compound

\section{Introduction}

Rooibos (Aspalathus linearis (Burm.f.) R. Dahlgren (Fabaceae)) is a leguminous shrub endemic to the Cape Floristic Region of South Africa. The species complex comprises several distinct growth forms, one of which (the Red type) has been cultivated since the 1930s for the production of rooibos herbal tea $[1,2]$. This tea is caffeine-free, low in tannins, high in volatile compounds and rich in polyphenols [3]. It is gaining international popularity, as an increasing body of literature provides scientific evidence for diverse health promoting properties of rooibos, including antiaging, anticancer, antispasmodic, antidiabetic and cardioprotective activities [4-8]. Many of these effects have been linked to the wide range of phenolic compounds produced by the plant $[9,10]$. Aspalathin, a C-glucosyl 
dihydrochalcone, is one of the major polyphenolic rooibos compounds, contributing $4-12 \%$ of the total dry matter of the plant $[11,12]$. Aspalathin is an extremely rare compound, which has so far only been detected in plants of the $A$. linearis species complex and in two populations of the sister species A. pendula [10] (but see [1] with regards to A. pendula). A. pendula is a rare plant species with a limited geographic distribution, used only by the local communities for making "yellow" tea. Therefore, aspalathin represents a potential marker compound for authentication and quality control of plant material and extracts prepared from A. linearis.

Fermentation of rooibos, traditionally performed to develop the characteristic red-brown color and slightly sweet flavor, involves chopping, bruising, "sweating" and subsequent drying of the plant material in the sun. The process is associated with a significant (over 90\%) reduction in aspalathin content [11,12], as it is oxidized to isoorientin and orientin [13]. Due to the higher polyphenol content, unfermented (green) rooibos is currently finding increased attention for the production of herbal tea and cosmetic products, and as a functional food ingredient [12,14]. So far, producers of green rooibos herbal tea mostly rely on visual inspection, using the red-brown discoloration of the plant material as an indicator for undesirable polyphenol oxidation and aspalathin degradation [15]. Chemical screening is restricted to analyses of the total polyphenol content and total antioxidant capacity, for which comparatively inexpensive and simple methods have been developed [12]. However, these methods do not allow conclusions on the aspalathin content. Exact aspalathin concentrations can be determined using various technologies such as HPLC, as well as Near Infrared and Raman spectroscopy $[15,16]$, but these methods require specialized equipment and expertise, and are therefore prohibitively expensive at high sample numbers.

Thin layer chromatography (TLC) represents a simple and easily scalable method for the detection of diverse compounds in plant extracts, herbal products and foods $[17,18]$. When paired with chromatogram densitometry or image analysis for compound quantification, TLC provides a cost-effective alternative to other detection methods (e.g., HPLC, NIS, RS) for high-throughput sample screening [18]. Marker compound screening using TLC-densitometry/image analysis requires the establishment of a protocol that ensures reliable compound separation and visualization. A TLC-method for analysis of flavonoids in tea samples, including rooibos tea, has been reported previously [19], however, aspalathin was not determined in that study. P-anisaldehyde is a universal derivatization agent which can be used to detect a wide range of nucleophilic compounds [20]. When investigating flavonoid fingerprints in Passiflora species, Birk et al. (2005) [21] found that p-anisaldehyde- $\mathrm{H} 2 \mathrm{SO} 4$ represents a suitable alternative derivatization reagent to the commonly used NP/PEG [22], which has also been applied by Ligor et al. (2008) [19]. Derivatization with p-anisaldehyde-H2SO4 not only produced distinct banding patterns, but also appeared to improve band resolution [21].

Here, we tested p-anisaldehyde for its ability to visualize aspalathin in thin layer chromatograms. We describe a simple, fast, cost-effective, sensitive and reproducible TLC method for the reliable detection of aspalathin in rooibos plant material and herbal tea samples. Aspalathin band purity was verified using HPLC-DAD and UPLC-MS, and TLC detection limits were determined using densitometry.

\section{Results and Discussion}

The species Aspalathus linearis currently comprises several diverse growth types [1], including prostrate shrublets (Southern and Northern sprouters); more or less densely branched shrubs (Nardouwsberg type, Grey sprouter, Nieuwoudtville sprouter, Wuppertal type); and erect, slender bushes (Red type, Black type, Tree type). The 34 plants analyzed in this study (Table 1, Supplementary Table S1, Figure S1) represent a subset from a total of 109 rooibos ecotypes with representatives from eight $A$. linearis growth types, including 28 commercial plants. 
Table 1. Description of rooibos ecotypes used in the analysis.

\begin{tabular}{|c|c|c|c|c|}
\hline Plant & Growth Type & Location & Region & $\begin{array}{l}\text { Aspalathin Concentration } \\
\text { Determined by HPLC-DAD } \\
\text { (g/100 g Plant Dry Weight) }\end{array}$ \\
\hline 1 & $\begin{array}{l}\text { Commercial } \\
\text { rooibos plant }\end{array}$ & $\begin{array}{l}\mathrm{S} 032^{\circ} 47^{\prime} 22^{\prime \prime} \\
\mathrm{E} 018^{\circ} 48^{\prime} 26^{\prime \prime}\end{array}$ & Clanwilliam & 7.36 \\
\hline 2 & $\begin{array}{l}\text { Commercial } \\
\text { rooibos plant }\end{array}$ & $\begin{array}{l}\mathrm{S} 031^{\circ} 43^{\prime} 17^{\prime \prime} \\
\mathrm{E} 019^{\circ} 07^{\prime} 29^{\prime \prime}\end{array}$ & Nieuwoudtville & 12.83 \\
\hline 3 & Grey sprouter & 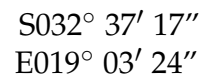 & Clanwilliam & 0.01 \\
\hline 4 & Grey sprouter & $\begin{array}{l}\mathrm{S} 032^{\circ} 37^{\prime} 17^{\prime \prime} \\
\mathrm{E} 019^{\circ} 03^{\prime} 24^{\prime \prime}\end{array}$ & Clanwilliam & 0.02 \\
\hline 5 & Grey sprouter & $\begin{array}{l}\mathrm{S} 032^{\circ} 37^{\prime} 17^{\prime \prime} \\
\mathrm{E} 019^{\circ} 03^{\prime} 24^{\prime \prime}\end{array}$ & Clanwilliam & 4.96 \\
\hline 6 & $\begin{array}{l}\text { Nardowsberg } \\
\text { type }\end{array}$ & 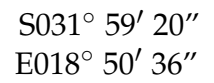 & Clanwilliam & 0.00 \\
\hline 7 & Black type & $\begin{array}{l}\mathrm{S} 032^{\circ} 24^{\prime} 38^{\prime \prime} \\
\mathrm{E} 019^{\circ} 00^{\prime} 51^{\prime \prime}\end{array}$ & Algeria & 0.04 \\
\hline 8 & Black type & $\begin{array}{l}\mathrm{S}_{0} 032^{\circ} 11^{\prime} 55^{\prime \prime} \\
\mathrm{E} 018^{\circ} 51^{\prime} 43^{\prime \prime}\end{array}$ & Algeria & 0.02 \\
\hline 9 & $\begin{array}{c}\text { Nieuwoudtville } \\
\text { sprouter }\end{array}$ & $\begin{array}{l}\mathrm{S} 031^{\circ} 42^{\prime} 55^{\prime \prime} \\
\mathrm{E} 019^{\circ} 07^{\prime} 40^{\prime \prime}\end{array}$ & Nieuwoudtville & 1.76 \\
\hline 10 & $\begin{array}{l}\text { Wypperthal } \\
\text { type }\end{array}$ & 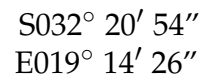 & Clanwilliam & 2.18 \\
\hline 11 & $\begin{array}{l}\text { Northern } \\
\text { sprouter }\end{array}$ & $\begin{array}{l}\mathrm{S} 032^{\circ} 13^{\prime} 29^{\prime \prime} \\
\mathrm{E} 019^{\circ} 07^{\prime} 52^{\prime \prime}\end{array}$ & Clanwilliam & 2.72 \\
\hline 12 & $\begin{array}{l}\text { Northern } \\
\text { sprouter }\end{array}$ & $\begin{array}{l}\mathrm{S} 032^{\circ} 13^{\prime} 32^{\prime \prime} \\
\mathrm{E} 019^{\circ} 07^{\prime} 55^{\prime \prime}\end{array}$ & Clanwilliam & 1.96 \\
\hline 13 & Black type & $\begin{array}{l}\mathrm{S} 031^{\circ} 45^{\prime} 48^{\prime \prime} \\
\mathrm{E} 019^{\circ} 07^{\prime} 54^{\prime \prime}\end{array}$ & Nieuwoudtville & 5.18 \\
\hline 14 & Black type & $\begin{array}{l}\mathrm{S} 031^{\circ} 59^{\prime} 21^{\prime \prime} \\
\mathrm{E} 018^{\circ} 50^{\prime} 35^{\prime \prime}\end{array}$ & Clanwilliam & 0.01 \\
\hline 15 & Tree type & 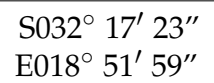 & Clanwilliam & 0.00 \\
\hline 16 & Tree type & $\begin{array}{l}\mathrm{S} 032^{\circ} 17^{\prime} 23^{\prime \prime} \\
\mathrm{E} 018^{\circ} 51^{\prime} 59^{\prime \prime}\end{array}$ & Clanwilliam & 0.00 \\
\hline
\end{tabular}

Classification of wild rooibos growth types was verified by Prof Van Wyk (University of Johannesburg).

\subsection{Aspalathin Visualization Using TLC}

Figure 1 shows TLC analyses conducted with methanol extracts from two aspalathin-producing commercial rooibos plants ( 1 and 2 ) and two wild rooibos plants ( 3 and 4 ) that produce only trace amounts of the compound (aspalathin concentrations were verified using HPLC-DAD; Table 1). The TLC banding patterns for plants 1 and 2 are representative for commercial rooibos plants: two purple bands at retention factor (RF) $0.53 \pm 0.04$ and $0.89 \pm 0.03$ (bands 1 and 3, respectively), which can vary in intensity; and a prominent orange-brown band at RF $0.46 \pm 0.03$ (band 4). Other bands, like the third purple band at RF $0.64 \pm 0.04$ (band 2) and the second orange band at RF $0.48 \pm 0.03$, appear depending on the plant genotype and TLC running conditions. 


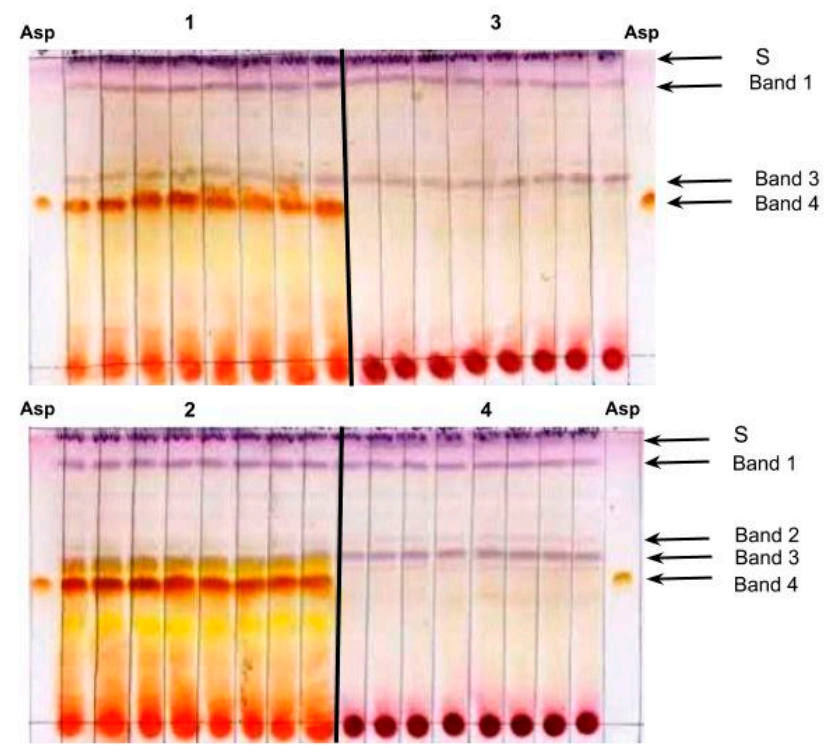

Figure 1. Repeatability test for the TLC method using four rooibos ecotypes with eight repeats. Plants 1 and 2 produce aspalathin, while plants 3 and 4 only produce trace amounts of this compound. Asp: aspalathin standard, S: solvent front.

The prominent orange-brown band 4 in samples 1 and 2 has the same RF value (0.46) and stains the same color as the aspalathin standard. The methanol extracts from plants 3 and 4 did not produce this band, and HPLC-DAD analysis verified only trace amounts of aspalathin (0.01 and 0.02 g/100 g $\mathrm{dw}$, respectively). To verify whether band 4 is produced by the aspalathin in the extracts from plants 1 and 2, we analyzed methanol extracts of silica scraps from the corresponding region on preparative TLC plates using HPLC-DAD (plants 1 and 2) and UPLC-MS (plant 1, 2, 13). For these samples, the silica scraps were found to contain aspalathin at very high purity (HPLC-DAD: supplemental Figure S2A and B; UPLC-MS: Figure 2). For plant 3, that produces only traces of aspalathin, silica scraps from the corresponding region of the preparative TLC plate appeared aspalathin-free (HPLC-DAD: supplemental Figure S2C).

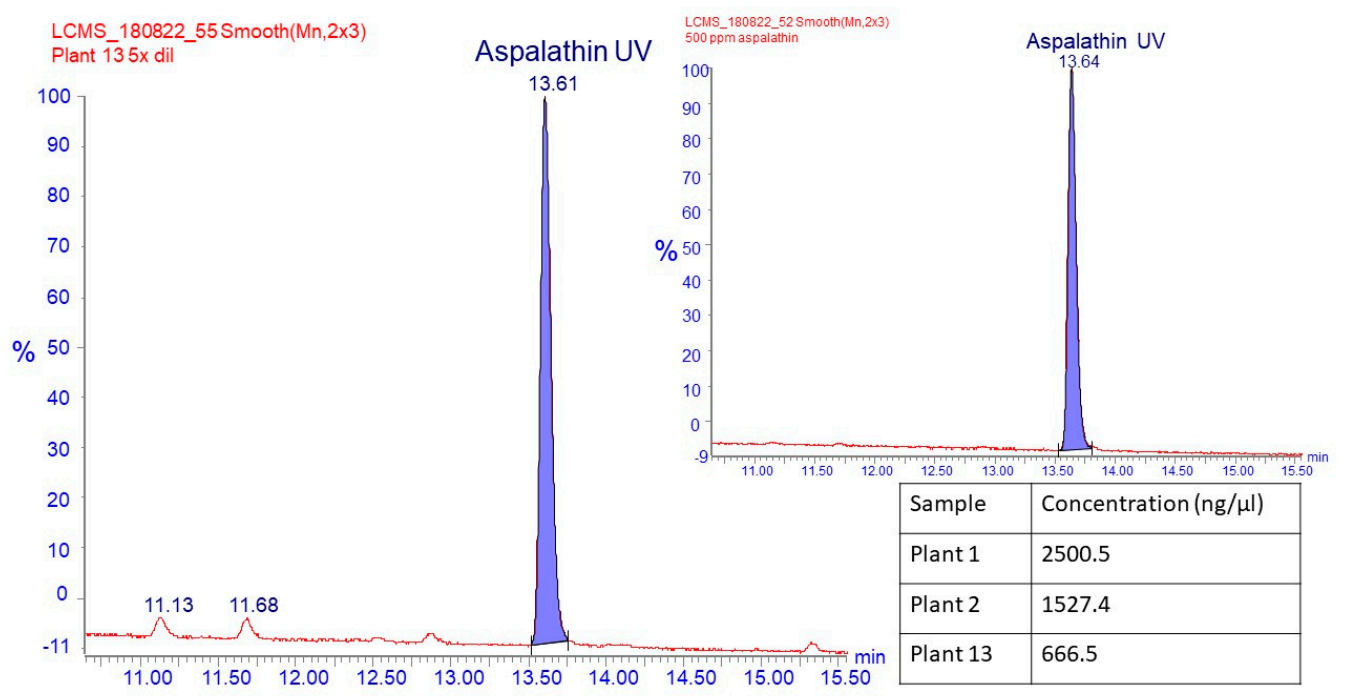

Figure 2. The chromatogram is the UPLC-MS chromatogram comparing TLC silica extracts of plants 1,2 and 13 at $\mathrm{RF}=0.46$ to the aspalathin standard (insert). The table shows the concentrations of aspalathin extracted by the preparative TLC extraction method. 
These analyses confirmed that the orange-brown band at RF 0.46 (band 4) was produced by the aspalathin present in the methanol extracts from the commercial and wild rooibos plants, i.e., that the described TLC method is suitable for visualization of this compound in plant extracts. This is the first report on using p-anisaldehyde for visualization of aspalathin in TLC analyses.

\subsection{Reproducibility and Sensitivity of the TLC Method}

To verify reproducibility, the two TLC plates depicted in Figure 1 were prepared by two different persons. The aspalathin bands were present in each of the eight technical repeats of extracts from plants 1 and 2, and absent in the eight technical repeats of extracts from plants 3 and 4 .

To determine the limits of detection (LOD) and quantification (LOQ) for pure aspalathin using the TLC method, we analyzed a sequence of defined aspalathin amounts, ranging from $50 \mathrm{ng}$ to $1000 \mathrm{ng}$. This standard curve analysis was replicated nine times, and aspalathin standards were reproducibly visualized at $200 \mathrm{ng}$ (Figure 3A; 1 replicate). Calculations conducted on the densitometric data of the nine replicates following the International Conference on Harmonization guidelines Q2 [23] (ICH, 2005) allowed determination of an LOD of $135.9 \mathrm{ng}$ and an LOQ of $411.9 \mathrm{ng}$ aspalathin when using the TLC method. To determine LOD and LOQ for aspalathin concentrations in plant samples, methanol extracts of the aspalathin negative plant number 4 were spiked with the compound and applied onto TLC plates to deliver between $400 \mathrm{ng}$ and $3 \mu \mathrm{g}$ of aspalathin per spot. This standard curve analysis was also replicated nine times (Figure 3B; 1 replicate). The aspalathin band is easily discernable at concentrations of $1 \mu \mathrm{g}$. Densitometric image analysis permits verification of aspalathin at much lower concentrations: $\mathrm{LOD}=178.7 \mathrm{ng}$ and $\mathrm{LOQ}=541.6 \mathrm{ng}$ aspalathin.
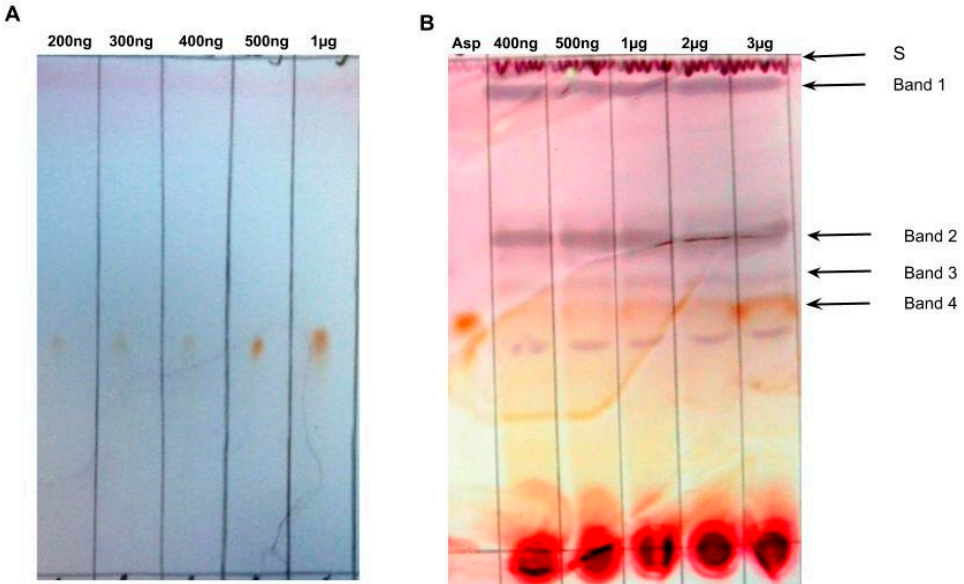

Figure 3. Lowest limit of detection for (A) pure aspalathin ( $n=9,1$ replicate shown), and (B) plant 3 (contains no aspalathin) spiked with different aspalathin concentrations ( $n=9,1$ replicate shown), using TLC. Asp: aspalathin standard, S: solvent front.

\subsection{TLC Analysis of Extracts from Wild Rooibos Ecotypes}

The TLC method was found suitable for visualization of aspalathin in plant extracts from non-commercial rooibos plants. Figure 4 shows the results for the TLC analyses conducted on 12 wild-growing rooibos ecotypes that represent different $A$. linearis growth types from distant locations in the Cederberg Mountain region (Table 1). Of the 12 samples evaluated by TLC, only six developed the orange-brown band at RF 0.46 (band 4), indicating the presence of aspalathin (Figure 4). For these six plants, HPLC-DAD analyses confirmed substantial aspalathin concentrations, ranging from 1.8 to $5.2 \mathrm{mg} / 100 \mathrm{~g}$ plant dry weight (Table 1). Extracts from the plants 6, 7, 8, 14, 15 and 16 did not produce the aspalathin band. HPLC-DAD analyses revealed that the extracts from plants 7, 8 and 14 had very low levels of aspalathin, and that the amounts loaded on the TLC plate (40.7 ng, $16.0 \mathrm{ng}$ and $5.8 \mathrm{ng}$ aspalathin, respectively) were below the calculated LOD (178.7 ng). The plants 5, 15 and $16 \mathrm{did}$ 
not produce aspalathin at concentrations measurable using the above HPLC-DAD method. The 12 samples showed a high variability in banding patterns, even between plants of the same growth type (e.g., Black type plants 7, 8, 14 and 13; Northern sprouters 11 and 12). Banding patterns were similar for plants from the same populations for the Black type plants 7 and 8 and for the Tree type plants 15 and 16, but not for the Grey sprouters (plants 3 and 4 did not produce measurable amounts of aspalathin; plant 5 did produce measurable amounts of aspalathin).

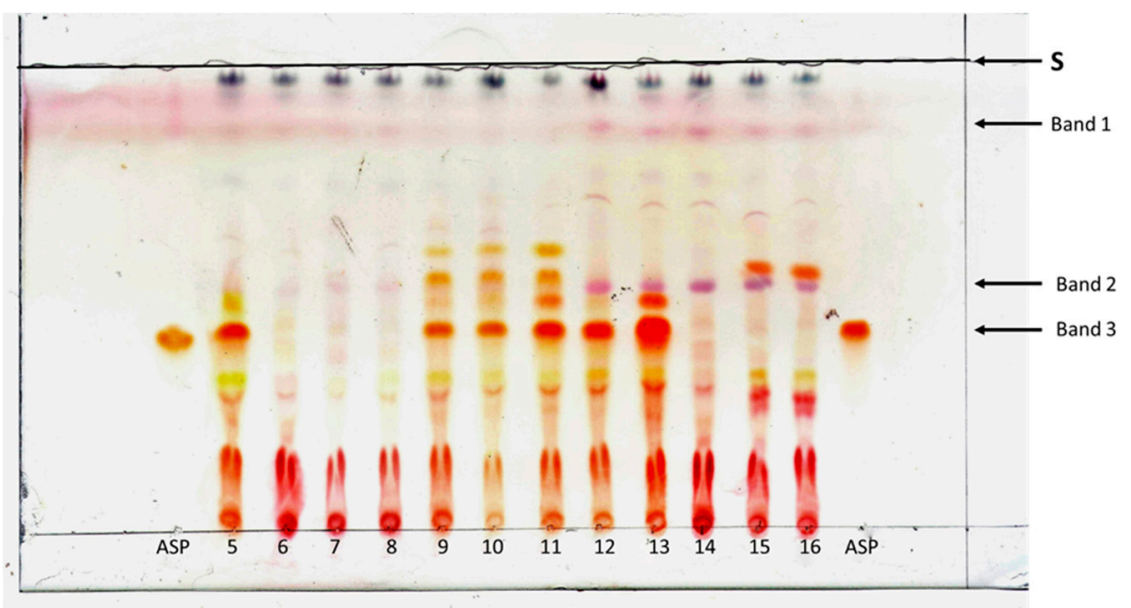

Figure 4. TLC analyses conducted on 12 wild-growing rooibos ecotypes representing different A. linearis growth types. An acidifier was included which significantly reduced tailing of the bands. Asp: aspalathin standard, S: solvent front.

Previous studies have shown that all eight described $A$. linearis growth types can produce aspalathin [10], though concentrations can vary substantially between populations and individual plants $[1,10]$. The samples from Southern sprouters and the Black type plants investigated in these studies contained only traces of aspalathin or appeared aspalathin-free, while most of the samples from Red type and Grey type plants were aspalathin rich. Our results are in line with these findings: three of the four Black type plants produced only trace amounts of aspalathin, and the Grey sprouters were either devoid of this compound or aspalathin rich (plant 5: $5 \mathrm{~g} / 100 \mathrm{~g}$ dry weight).

The variability in aspalathin production in wild rooibos plants contrasted with the consistent banding patterns observed with extracts from commercial rooibos plants, where the aspalathin band was always clearly visible (Figure 1, Supplementary Figure S1). The commercially grown rooibos originates from Red type plants that were selected in the 1930s by Dr. P. le Fras Nortier, who favored a bushy upright growth form (which simplified harvest), high growth rates and distinctive taste [12]. This selection may have favored aspalathin-rich plants, which could explain the more uniform banding patterns and consistent visualization of the aspalathin band in our extracts from commercial rooibos plants. Joubert and de Beer (2011) [12] report on variability in aspalathin contents between 21 commercial rooibos plants and mention significant seasonal effects, but the reported concentrations of this compound were never below $2.33 \mathrm{~g}$ aspalathin/100 g plant dry matter, as verified by HPLC-DAD.

\subsection{TLC Analysis of Extracts from Commercial Rooibos Herbal Tea Samples}

Green rooibos herbal tea was first introduced in the 1990s as a project of the Agricultural Research Council of South Africa [12] and gained popularity due to higher flavonoid concentrations (including aspalathin) in comparison to fermented rooibos herbal tea [24,25]. To determine whether differences in aspalathin concentrations between green and fermented rooibos can be visualized with the described TLC method, we sampled and analyzed two commercially available green and six fermented rooibos herbal tea brands. Both green rooibos herbal tea brand samples (Figure 5, lanes A and B) had more prominent and darker aspalathin bands than the five fermented rooibos herbal tea brand samples 
(Figure 5, lanes C-H). HPLC-DAD confirmed that aspalathin was present at much higher concentrations in the green rooibos herbal tea extracts than in the fermented rooibos herbal tea extracts (Table 2), mirroring the TLC results. Therefore, our method can be used to distinguish between green and fermented rooibos herbal tea.

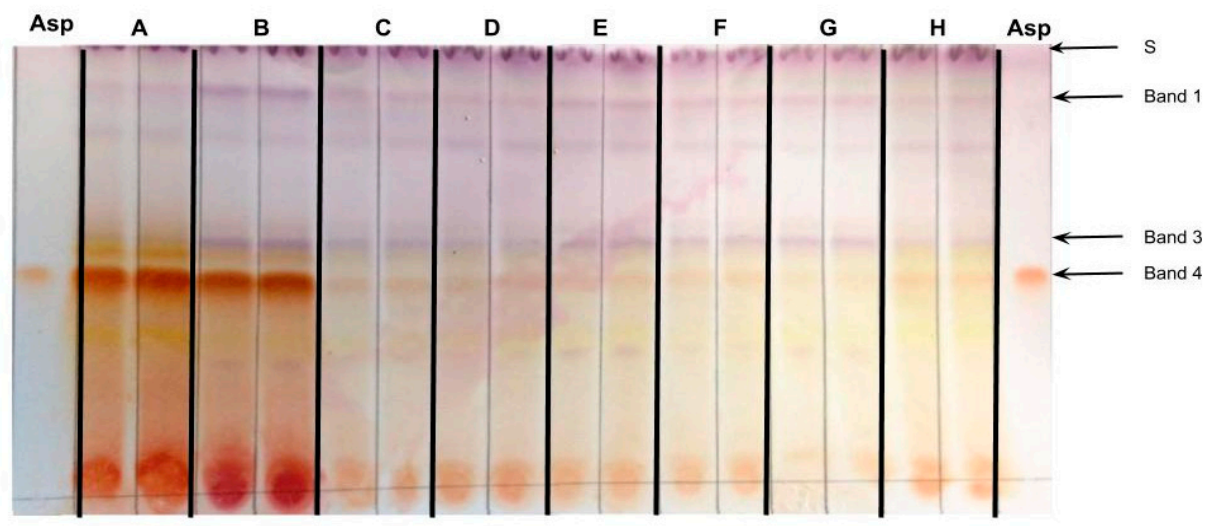

Figure 5. TLC analysis of samples from A) two green (lanes A and B) and six fermented (lanes $\mathrm{C}-\mathrm{H})$ rooibos herbal tea brands. Analysis was performed in duplicate. Asp: aspalathin standard, S: solvent front.

Table 2. Aspalathin concentrations (g/100 g plant dry weight) determined by HPLC-DAD in green and fermented rooibos herbal tea extracts.

\begin{tabular}{ccc}
\hline Tea & Herbal Tea Type & Aspalathin Concentration (g/100 g Tea Leaves) \\
\hline A & Green & 14.47 \\
\hline B & Green & 14.20 \\
\hline C & Fermented & 0.20 \\
\hline D & Fermented & 0.51 \\
\hline E & Fermented & 0.50 \\
\hline F & Fermented & 1.04 \\
\hline G & Fermented & 0.84 \\
\hline H & Fermented & 0.99 \\
\hline
\end{tabular}

\subsection{Summary}

In this study, we describe a TLC method that allows the detection of aspalathin in rooibos plant material and rooibos herbal tea samples using p-anisaldehyde- $\mathrm{H}_{2} \mathrm{SO}_{4}$ as a derivatization reagent. It reproducibly visualized aspalathin in commercial rooibos plants and permitted sorting of high and low / zero aspalathin producers among the wild rooibos ecotypes. It could therefore be applied for low-cost high-throughput screening of rooibos ecotypes for targeted plant selection. For plant sample analyses, we used mostly aspalathin-rich leaf material, minimized aspalathin degradation by flash-freezing samples in the field and maintaining them at $-80{ }^{\circ} \mathrm{C}$, and maximized compound extraction by powdering the samples. Tea samples were analyzed without such pre-processing steps to reflect possible differences in tea quality (as affected by leaf/stem content and particle size due to different sieve sizes). The TLC method allowed differentiation between green and fermented tea samples and could therefore serve for cost-effective high-throughput quality assessment and authentication of green rooibos herbal teas. Aspalathin concentrations in fermented rooibos herbal tea samples were close to the detection limit of the TLC method. For fermented rooibos herbal tea samples, optimization of the TLC method (such as powdering of the leaf material, increased biomass, extraction 
procedures) is advisable. Furthermore, the TLC method can be adapted for High Performance TLC, which should improve reproducibility and sensitivity.

\section{Materials and Methods}

\subsection{Reagents and Materials}

All solvents (analytical grade) were acquired from Merck (Kenilworth, NJ, USA). Aspalathin was obtained from Chromadex Chemicals (California, CA, USA), and p-anisaldehyde (4-methoxybenzaldehyde) was procured from Sigma-Aldrich (St. Louis, MO, USA).

\subsection{Plant and Tea Material}

Morphologically distinct rooibos ecotypes, including commercially farmed and wild rooibos plants representing eight $A$. linearis growth forms, were sampled in spring (October/November) in the Clanwilliam (Western Cape), Wupperthal (Western Cape) and Nieuwoudtville (Northern Cape) regions in the Cederberg Mountains, South Africa (Table 1, Supplementary Table S1). Up to $100 \mathrm{~g}$ of rooibos leaves and stems were collected from four sides of each plant, flash frozen in the field with liquid nitrogen, transported on dry ice, and stored at $-80^{\circ} \mathrm{C}$. For the analysis of commercially available teas, different brands of green and fermented rooibos herbal tea were purchased from local supermarkets.

\subsection{Plant, Rooibos Herbal Tea Extract Preparation}

Plant samples were freeze dried and ground to a fine powder using a coffee grinder. Then, $50 \mathrm{mg}$ of plant material was extracted in $5 \mathrm{~mL}$ methanol. Tea samples remained unprocessed (i.e., were not ground), and $500 \mathrm{mg}$ was extracted in $5 \mathrm{~mL}$ methanol. All samples were rotated at $40 \mathrm{rpm}$ on a Benchmark rotating mixer (Benchmark Scientific, Edison, NJ, USA) at $4{ }^{\circ} \mathrm{C}$ for $24 \mathrm{~h}$.

\subsection{Thin-Layer Chromatography (TLC)}

The TLC method was adapted from Ligor et al. (2008) [19]. First, a Twin Trough TLC Chamber for $20 \times 10 \mathrm{~cm}$ TLC plates (CAMAG, Switzerland) was pre-equilibrated for $30 \mathrm{~min}$ with solvent vapors by lining the chamber with filter paper moistened with mobile phase solution (acetone-chloroform-water; 80:20:10; $v / v / v$ ). For TLC, $10 \mu \mathrm{L}$ of each plant sample, $15 \mu \mathrm{l}$ of each tea sample, and $10 \mu \mathrm{L}$ of the methanol-dissolved aspalathin standard $(100 \mathrm{ng} / \mu \mathrm{L})$ were applied manually as spots to silica gel $60 \mathrm{~F} 25420 \times 20 \mathrm{~cm}$ aluminum plates (Merk) using a $200 \mu \mathrm{L}$ pipet tip. The addition of $10 \mu \mathrm{L}$ of formic acid per $\mathrm{ml}$ of methanol extract just before the TLC analysis was found to substantially reduce tailing of the samples and the aspalathin standard. TLC plates were developed using the mobile phase solution described above. For derivatization, the TLC plates were sprayed until saturation with a p-anisaldehyde solution ( $0.5 \%$ p-anisaldehyde, $1 \%$ sulfuric acid (95\% to 99\%) and $98.5 \%$ glacial acetic acid; $v / v / v$ ) using an air compressor with air brush (Aircraft Pneumatic System, AS18); and subsequently exposed to heat using a heat-gun $(1750 \mathrm{~W}$, Black and Decker) until the bands became visible. RF values for selected bands were calculated using TLC results from 15 plant samples analyzed on different TLC plates. The retention factor (RF) was calculated as the ratio of the travel distance the band to the travel distance of the solvent front.

Preparative TLC analyses were performed following the above procedures, using $50 \mu \mathrm{L}$ of plant extract per $3 \mathrm{~cm}$ TLC plate. After development, the silica in the region of the aspalathin standard was scraped off and extracted with $1 \mathrm{~mL}$ of methanol by vortexing for $20 \mathrm{~min}$ at room temperature. The resulting methanol extract was subsequently filtered through a $0.22 \mu \mathrm{m}$ filter.

\subsection{Image Analysis for Determination of $L O D$ and $L O Q$}

The TLC plates were scanned using an I-Sensys MF41010 Scanner (Canon) to obtain chromatographic images and densitometry of images was conducted using ImageJ [26]. 
To determine limits of detection (LOD) and quantitation (LOQ), we conducted TLC analyses with methanol-dissolved commercial aspalathin (200 ng, $300 \mathrm{ng}, 400 \mathrm{ng}, 500 \mathrm{ng}$ and $1000 \mathrm{ng}$ aspalathin per spot) as well as with aspalathin-spiked plant extracts from an aspalathin-negative rooibos ecotype (400 ng, $500 \mathrm{ng}, 1 \mu \mathrm{g}, 2 \mu \mathrm{g}$ and $3 \mu \mathrm{g}$ aspalathin per spot). The densitometric measurements were used to calculate LOD and LOQ following the International Conference on Harmonization (ICH) guidelines Q2 (ICH, 2005). Statistical analyses were performed in Excel (Microsoft) using the following formulas: $\mathrm{LOD}=3.3 \times($ standard deviation of the response $) /($ slope of calibration curve); LOQ $=10 \times($ standard deviation of the response)/(slope of calibration curve).

\subsection{High Performance Liquid Chromatography (HPLC-DAD)}

HPLC analysis of rooibos methanol extracts was adapted from Bramati et al. (2002) [27] using the Agilent 1200 series HPLC system coupled to a diode array detector (DAD) (Santa, Clara, CA, USA) with a Nucleosil 120-5C18 column $15 \mathrm{~cm} \times 4.6 \mathrm{~mm}(5 \mu \mathrm{M}$, Sigma Aldrich). Column temperature was maintained at $21^{\circ} \mathrm{C}$. Mobile phase A was $300 \mu \mathrm{L} / \mathrm{L}$ trifluoracetic acid in water and mobile phase B was $300 \mu \mathrm{L} / \mathrm{L}$ trifluoracetic acid in methanol. A sample volume of $20 \mu \mathrm{L}$ was injected. A constant flow rate of $1 \mathrm{~mL} / \mathrm{min}$ was maintained and the gradient elution was performed as follows: $5 \% \mathrm{~B}$ for $5 \mathrm{~min}$, linear increase to $80 \%$ B over $20 \mathrm{~min}$, decrease to $35 \%$ B over $3 \mathrm{~min}, 35 \%$ B for $2 \mathrm{~min}$, re-equilibration to $5 \%$ B. Acquisition was set at $287 \mathrm{~nm}$ for aspalathin detection. Peak areas of the samples were compared to a $20 \mathrm{ng} / \mu \mathrm{L}$ aspalathin standard to determine aspalathin concentrations of the samples.

\subsection{UPLC-MS Analysis}

To confirm the purity of the aspalathin peaks obtained using HPLC-DAD, selected samples were also analyzed using high precision UPLC-MS. This analysis was performed at the Central Analytical Facility of Stellenbosch University (WC, South Africa) using a Waters Acquity ultra performance liquid chromatograph (UPLC) coupled to a photo diode array and a Waters Synapt G2 Quadrupole time-of-flight (QTOF) mass spectrometer (MS) equipped with an electrospray ionization source (Waters, Milford, MA, USA). Electrospray ionization was used in negative mode with a cone voltage of 15 $\mathrm{V}$, desolvation temperature of $275^{\circ} \mathrm{C}$, desolvation gas (nitrogen) at $650 \mathrm{~L} / \mathrm{hr}$, and the rest of the MS settings optimized for best resolution and sensitivity. Data, acquired in MSE mode, included a low collision energy scan (6 V) from $\mathrm{m} / \mathrm{z} 150$ to 1500; and a high collision energy scan from $\mathrm{m} / \mathrm{z} 40$ to 1500. The high collision energy scan was performed using a collision energy ramp (30 to $60 \mathrm{~V}$ ). The photo diode array detector was set to scan from 220 to $600 \mathrm{~nm}$. Sodium formate was used for calibration and leucin encephalin was infused in the background as lock mass for accurate mass determinations. Separation was achieved on a Waters HSS T3, $2.1 \times 100 \mathrm{~mm}, 1.7 \mu \mathrm{m}$ column at a column temperature of $60{ }^{\circ} \mathrm{C}$ and a constant flow rate of $0.25 \mathrm{~mL} / \mathrm{min}$. An injection volume of $3 \mu \mathrm{l}$ was used. Mobile phase A was $0.1 \%$ formic acid in water, and mobile phase B was acetonitrile. The gradient elution was performed as follows: 100\% A for $1 \mathrm{~min}$, linear increase of B to 50\% over $21 \mathrm{~min}$, increase of B to $100 \%$ over $1 \mathrm{~min}$, wash step at $100 \%$ B for $1.5 \mathrm{~min}$, and re-equilibration to initial conditions for $4.5 \mathrm{~min}$. The mass and concentration of aspalathin contained in the samples were compared to an aspalathin standard and an aspalathin standard curve $(10 \mathrm{mg} / \mathrm{L}, 100 \mathrm{mg} / \mathrm{L}, 200 \mathrm{mg} / \mathrm{L}$ and $500 \mathrm{mg} / \mathrm{L})$.

Supplementary Materials: The Supplementary Materials are available online. Figure S1: A subset from a total of 109 rooibos ecotypes analyzed with the described TLC method. Plants 17-25,26,29-31,34 = Commercial; plant 27,28,32,33 = wild-growing. S: solvent front. Figure S2: HPLC-DAD and UPLC-MS chromatograms of the preparative TLC extracts at $\mathrm{RF}=0.46$ suspected to contain aspalathin. The chromatograms $\mathrm{A}$ and $\mathrm{B}$ are TLC silica extracts from plants 1 and 2, verifying the presence of aspalathin at retention time $=9.1 \mathrm{~min}($ arrow $)$. The chromatogram $C$ is a silica extract at $R F=0.46$ from a TLC of an aspalathin negative plant 3 , verifying no aspalathin (arrow) at the equivalent position., Table S1: Description of sampled rooibos subset.

Author Contributions: Conceptualization, E.A.S., J.M., M.L.R.-H., U.H. and W.W.; Methodology, E.A.S., F.R., J.M., M.L.R.-H., U.H. and W.W.; Investigation, E.A.S., F.R., W.W. and Y.M.; Validation, E.A.S., F.R., W.W. and Y.M.; Formal Analysis, F.R. and W.W.; Resources, F.R., J.M. and M.L.R.-H. and U.H.; Writing-Original Draft Preparation, E.A.S., U.H. and W.W.; Writing-Review \& Editing, E.A.S., J.M., M.L.R.-H., U.H. and W.W.; Visualization, E.A.S., 
W.W. and U.H.; Supervision, J.M., F.R., M.L.R.-H., U.H. and W.W.; Project Administration, U.H., J.M., and M.L.R.-H.; Funding Acquisition, U.H., J.M., and M.L.R.-H.

Funding: This research was funded by the National Research Foundation (NRF), grant number CSUR150714125961. Opinions expressed, and conclusions arrived at, are those of the author and are not necessarily to be attributed to the NRF.

Acknowledgments: For assistance in plant collection we herewith would like to sincerely thank Rooibos LTD, specifically Johan Brand and Jan Perang; the Fair Wupperthal Rooibos Cooperative, specifically Edgar Valentyn; Darren Engelbrecht and Ruth Cuttings from the Department of Agriculture, Land Reform, and Rural Development (Northern Cape); as well as the following rooibos farmers: Adam \& Johan Christiaans, Annes \& Johan Brand, Carina Marais, Chrisjan Fortuin, Collin Fredericks, Danie Carstens, Davie Kritzinger, Deon Lambrechts, Edgar Valentyn, Frances Nel, Frans Du Plessis, Frans Kotze, Fritz Hanekam, Gerrie Olivier, Ian Brand, Johan \& Rita Bruwer, Niklaas Kupido, Willie Nel. We are sincerely grateful to B.E. Van Wyk for assistance in the classification of the wild rooibos plants. We gratefully acknowledge Central Analytical Facility, Stellenbosch University for the UPLC-MS analysis.

Conflicts of Interest: The authors declare no conflict of interest.

\section{References}

1. Van Wyk, B.E.; Gorelik, B. The history and ethnobotany of Cape herbal teas. S. Afr. J. Bot. 2017, 110, 18-38. [CrossRef]

2. Van Heerden, F.R.; Van Wyk, B.E.; Viljoen, A.M.; Steenkamp, P.A. Phenolic variation in wild populations of Aspalathus linearis (rooibos tea). Biochem. Syst. Ecol. 2003, 31, 885-895. [CrossRef]

3. Joubert, E.; Gelderblom, W.C.A.; Louw, A.; de Beer, D. South African herbal teas: Aspalathus linearis, Cyclopia spp. and Athrixia phylicoides-A review. J. Ethnopharmacol. 2008, 119, 376-412. [CrossRef] [PubMed]

4. Hattingh, A.; de Venter, M.; Joubert, E.; Koekemoer, T. The anti-ageing potential of rooibos (Aspalathus linearis): Preserving preadipocyte function. Planta Med. 2014, 80, P2P11. [CrossRef]

5. Marnewick, J.; Joubert, E.; Joseph, S.; Swanevelder, S.; Swart, P.; Gelderblom, W. Inhibition of tumour promotion in mouse skin by extracts of rooibos (Aspalathus linearis) and honeybush (Cyclopia intermedia), unique South African herbal teas. Cancer Lett. 2005, 112, 193-202. [CrossRef]

6. Gilani, A.H.; Khan, A.U.; Ghayur, M.N.; Ali, S.F.; Herzig, J.W. Antispasmodic effects of rooibos tea (Aspalathus linearis) is mediated predominantly through $\mathrm{K}+-$ channel activation. Basic Clin. Pharmacol. Toxicol. 2006, 99, 365-373. [CrossRef]

7. Muller, C.J.F.; Joubert, E.; Pheiffer, C.; Ghoor, S.; Sanderson, M.; Chellan, N.; Fey, S.J.; Louw, J. Z-2-( $\beta$-d-glucopyranosyloxy)-3-phenylpropenoic acid, an $\alpha$-hydroxy acid from rooibos (Aspalathus linearis) with hypoglycemic activity. Mol. Nutr. Food Res. 2013, 57, 2216-2222. [CrossRef]

8. Johnson, R.; de Beer, D.; Dludla, P.V.; Ferreira, D.; Muller, C.J.F.; Joubert, E. Aspalathin from rooibos (Aspalathus linearis): A bioactive C-glucosyl dihydrochalcone with potential to target the metabolic syndrome. Planta Med. 2018, 84, 568-583. [CrossRef]

9. Walters, N.A.; de Villiers, A.; Joubert, E.; de Beer, D. Phenolic profiling of rooibos using off-line comprehensive normal phase countercurrent chromatography reversed phase liquid chromatography. J. Chromatogr. A 2017, 1490, 102-114. [CrossRef]

10. Stander, M.A.; Van Wyk, B.E.; Taylor, M.J.C.; Long, H.S. Analysis of phenolic compounds in rooibos tea (Aspalathus linearis) with a comparison of flavonoid-based compounds in natural populations of plants from different regions. J. Agric. Food Chem. 2017, 65, 10270-10281. [CrossRef]

11. Schulz, H.; Joubert, E.; Schütze, W. Quantification of quality parameters for reliable evaluation of green rooibos (Aspalathus linearis). Eur. Food Res. Technol. 2003, 216, 539-543. [CrossRef]

12. Joubert, E.; de Beer, D. Rooibos (Aspalathus linearis) beyond the farm gate: From herbal tea to potential phytopharmaceutical. S. Afr. J. Bot. 2011, 77, 869-886. [CrossRef]

13. Krafczyk, N.; Heinrich, T.; Porzel, A.; Glomb, M.A. Oxidation of the dihydrochalcone aspalathin leads to dimerization. J. Agric. Food Chem. 2009, 57, 6838-6843. [CrossRef] [PubMed]

14. Miller, N.; De Beer, D.; Aucamp, M.; Malherbe, C.J.; Joubert, E. Inulin as microencapsulating agent improves physicochemical properties of spray-dried aspalathin-rich green rooibos (Aspalathus linearis) extract with $\alpha$-glucosidase inhibitory activity. J. Funct. Foods 2018, 48, 400-409. [CrossRef]

15. Joubert, E.; Schultz, H. Production and quality aspects of rooibos tea and related products: A review. J. Appl. Bot. Food Qual. 2012, 80, 138-144. 
16. Joubert, E.; Ferreira, D. Antioxidants of rooibos tea-A possible explanation for its health promoting properties? SA J. Food Sci. Nutr. 1996, 8, 79-82.

17. Mohammad, A.; Bhawani, S.A.; Sharma, S. Analysis of herbal products by thin-layer chromatography: A review. Int. J. Pharma Bio Sci. 2010, 1, PS16.

18. Sherma, J.; Rabel, F. A review of thin layer chromatography methods for determination of authenticity of foods and dietary supplements. J. Liq. Chromatogr. Relat. Technol. 2018, 41, 645-657. [CrossRef]

19. Ligor, M.; Kornyšova, O.; Maruška, A.; Buszewski, B. Determination of flavonoids in tea and rooibos extracts by TLC and HPLC. J. Planar Chromatogr. 2008, 21, 355-360. [CrossRef]

20. Jork, H.; Funk, W.; Fischer, W.; Wimmer, H.; Jork, H.; Funk, W.; Fischer, W.; Wimmer, H. Thin-Layer Chromatography: Reagents and Detection Methods; -VCH: Weinheim, Germany, 1990; Volume 1a, ISBN 3527278346.

21. Birk, C.D.; Provensi, G.; Gosmann, G.; Reginatto, F.H.; Schenkel, E.P. TLC fingerprint of flavonoids and saponins from Passiflora species. J. Liq. Chromatogr. Relat. Technol. 2005, 28, 2285-2291. [CrossRef]

22. Fried, B.; Sherma, J. Practical Thin-Layer Chromatography: A Multidisciplinary Approach; Taylor \& Francis: Didcot, UK, 1996; ISBN 9780849326608.

23. ICH. Validation of Analytical Procedures: Text and Methodolody Q2(R1); ICH: Geneva, Switzerland, 2005.

24. Walters, N.A.; de Villiers, A.; Joubert, E.; de Beer, D. Improved HPLC method for rooibos phenolics targeting changes due to fermentation. J. Food Compos. Anal. 2017, 55, 20-29. [CrossRef]

25. Joubert, E.; De Beer, D. Antioxidants of rooibos beverages. Role of plant composition and processing. In Processing and Impact on Antioxidants in Beverages; Elsevier: New York, NY, USA, 2014; ISBN 9780124047389.

26. Schneider, C.A.; Rasband, W.S.; Eliceiri, K.W. NIH Image to ImageJ: 25 years of image analysis. Nat. Methods 2012, 9, 671. [CrossRef] [PubMed]

27. Bramati, L.; Minoggio, M.; Gardana, C.; Simonetti, P.; Mauri, P.; Pietta, P. Quantitative characterization of flavonoid compounds in rooibos tea (Aspalathus linearis) by LC-UV/DAD. J. Agric. Food Chem. 2002, 50, 5513-5519. [CrossRef] [PubMed]

Sample Availability: The authors can be contacted for support if plant material is required for research purposes.

(C) 2019 by the authors. Licensee MDPI, Basel, Switzerland. This article is an open access article distributed under the terms and conditions of the Creative Commons Attribution (CC BY) license (http:// creativecommons.org/licenses/by/4.0/). 